\title{
In-flight performance of the polarization modulator in the CLASP rocket experiment
}

S. Ishikawa*, T. Shimizu (ISAS/JAXA), R. Kano, T. Bando, R. Ishikawa, G. Giono (NAOJ), D. Beabout, B. Beabout (NASA/MSFC),

S. Nakayama, T. Tajima (Mitsubishi Precision Co.,), and the CLASP team

※ s.ishikawa@solar.isas.jaxa.jp

\section{Abstract}

We developed a polarization modulation unit (PMU), a motor system to rotate a waveplate continuously. We applied this PMU for the Chromospheric Lyman-alpha SpectroPolarimeter (CLASP), a sounding rocket experiment to observe the linear polarization of the Lyman-alpha emission (121.6 nm vacuum ultraviolet) from the upper chromosphere and transition region of the Sun with a high polarization sensitivity of $0.1 \%$ for the first time and investigate the vector magnetic field. Rotation non-uniformity of the waveplate causes error in the polarization degree (i.e. scale error) and crosstalk between Stokes components. In the ground tests, we confirmed that PMU has superior rotation uniformity. CLASP was successfully launched on September 3, 2015, and PMU functioned well as designed. PMU achieved a good rotation uniformity during the flight and the high precision polarization measurement of CLASP was successfully achieved.

\section{Polarization observation by CLASP}

We performed the Chromospheric Lyman-alpha Spectropolarimeter (CLASP) sounding rocket experiment to observe the linear polarization of the Lyman-alpha light (Vacuum Ultraviolet, $\lambda=121.6 \mathrm{~nm}$ ) from the Sun with a high sensitivity and investigate magnetic fields of the upper chromosphere and transition region which were technically difficult so far. CLASP rotates the polarization angle of incident photons continuously by a rotating half waveplate with a period of $4.8 \mathrm{~s}$ per rotation, and extracts polarization component with a certain angle. By the amplitude and phase of modulated time profile (polarization modulation), CLASP obtains polarization degree and angle.

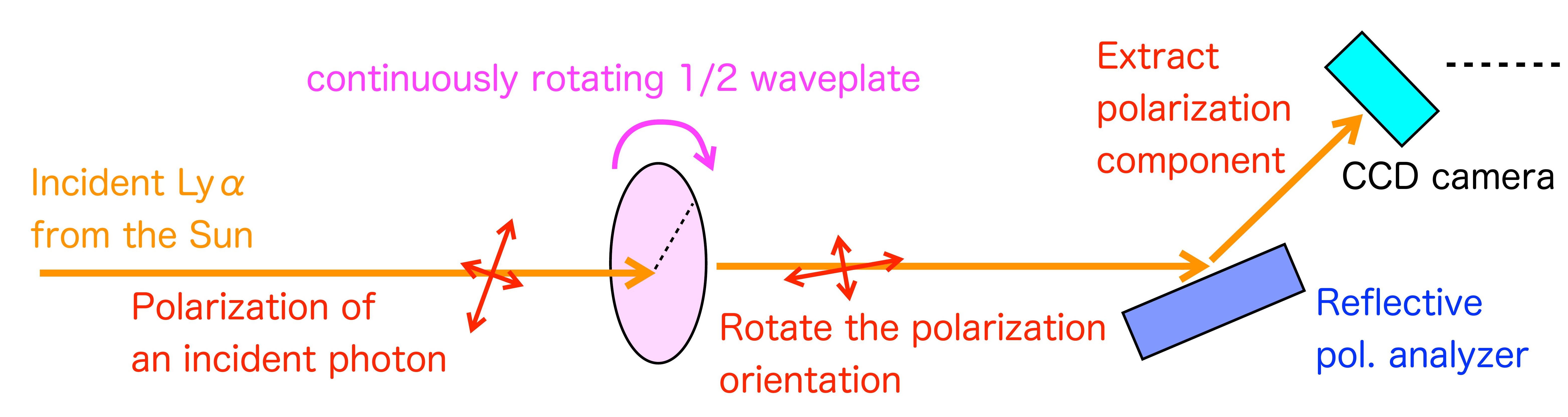

If the rotation is not uniform, polarization degree and angle are not correctly estimated (scale error and crosstalk). These causes the errors in magnetic field estimation, and high rotation uniformity is required. We used a polarization modulation unit (PMU) we developed to rotate the waveplate uniformly for CLASP. In the ground test, it was confirmed that scale error and crosstalks are as low enough as $<0.01 \%$ (Ishikawa et al. 2015, Sol. Phys.).
16 observation per every rotation $\rightarrow$ Polarization degree

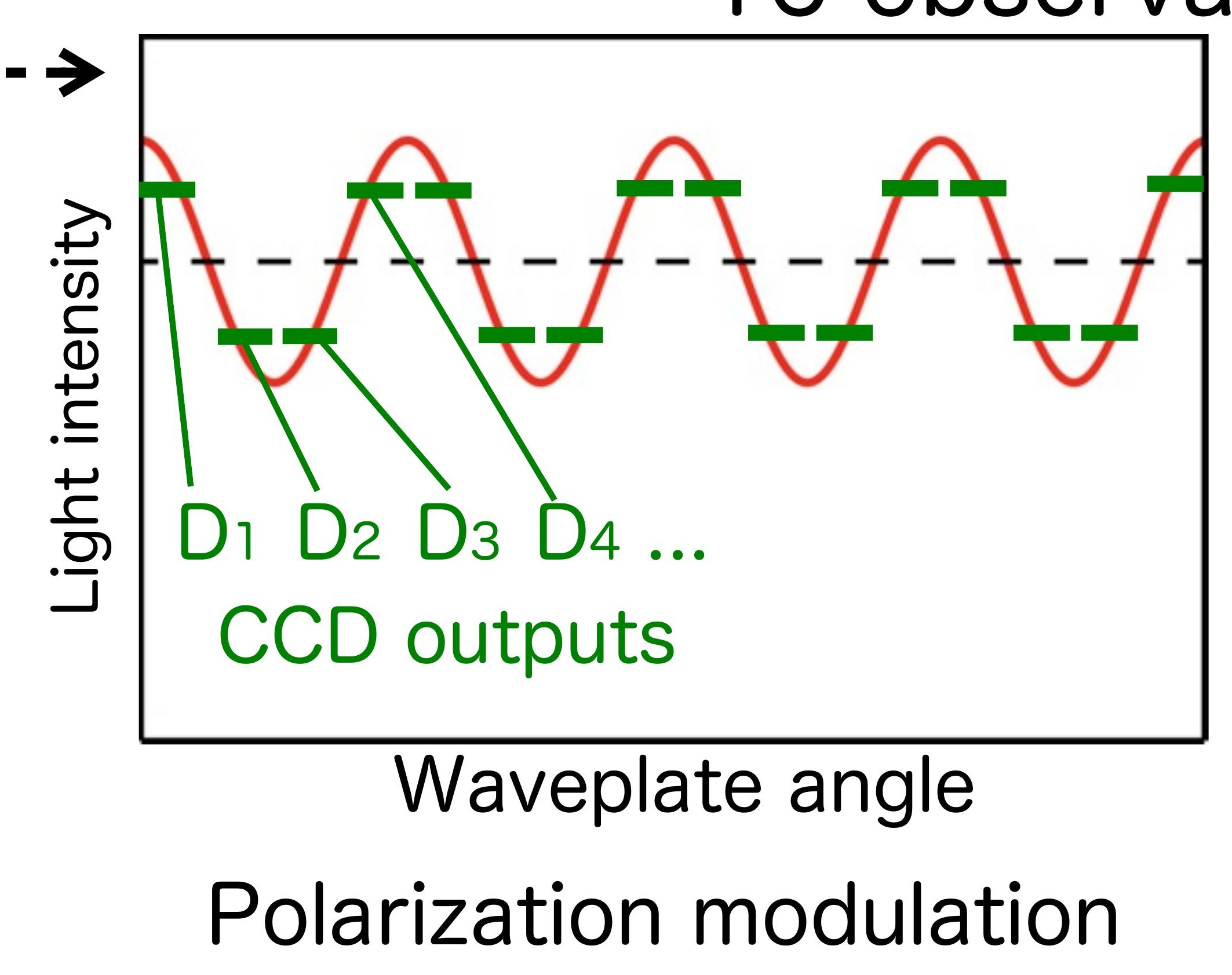

Polarization degree
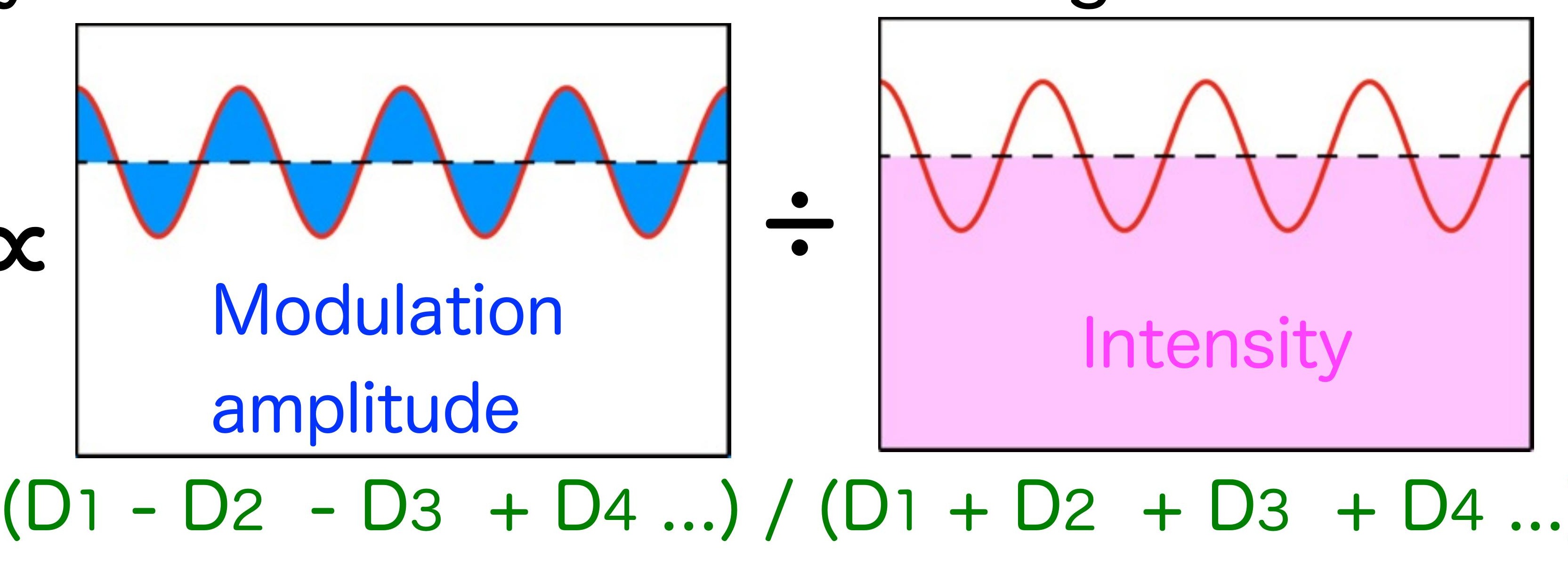

Polarization modulation

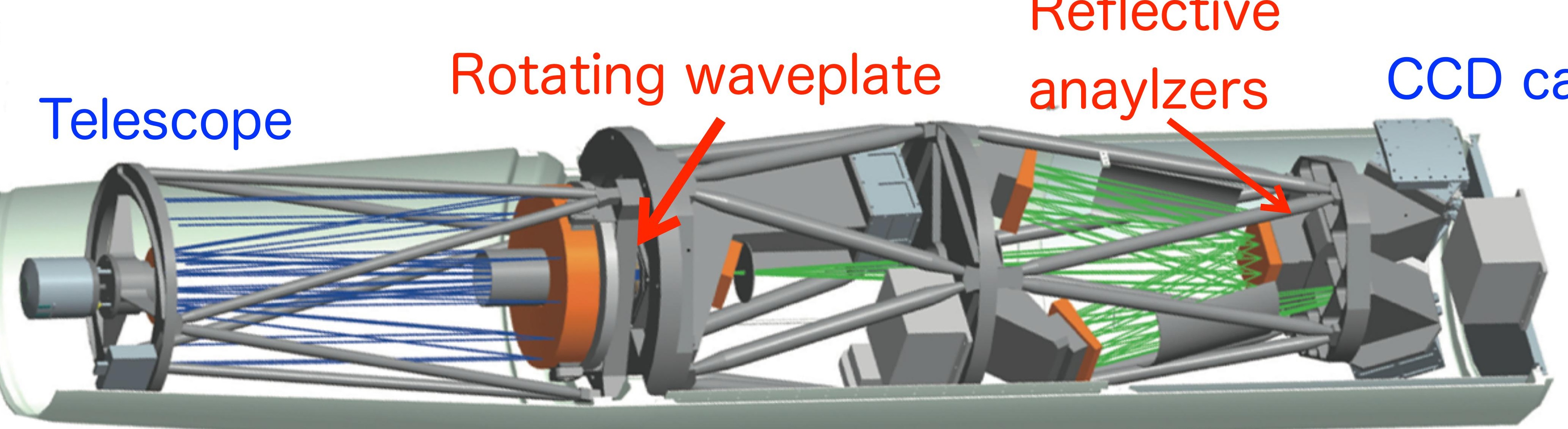



\section{Vibration tests and launch}

We performed vibration tests for the driver circuit of PMU which was newly developed for CLASP, and whole CLASP spectropolarimeter at ISAS. It was confirmed that the performance did not change after those tests.

After that, CLASP spectropolarimeter shipped to the United States and launched from the White Sands Missle Range on September 3, 2015. The launch and spectropolarimetric observation of the Sun were successfully performed.

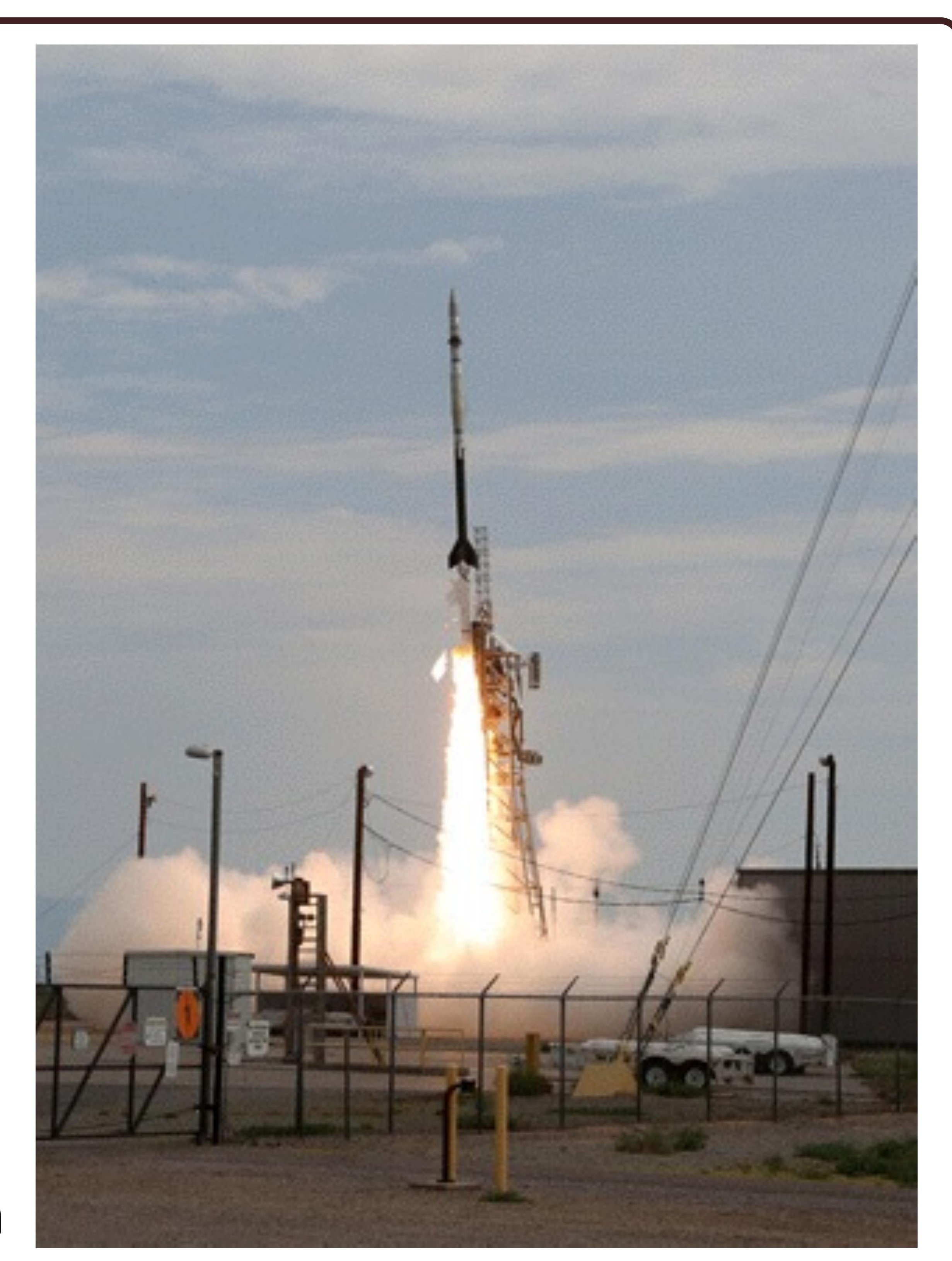

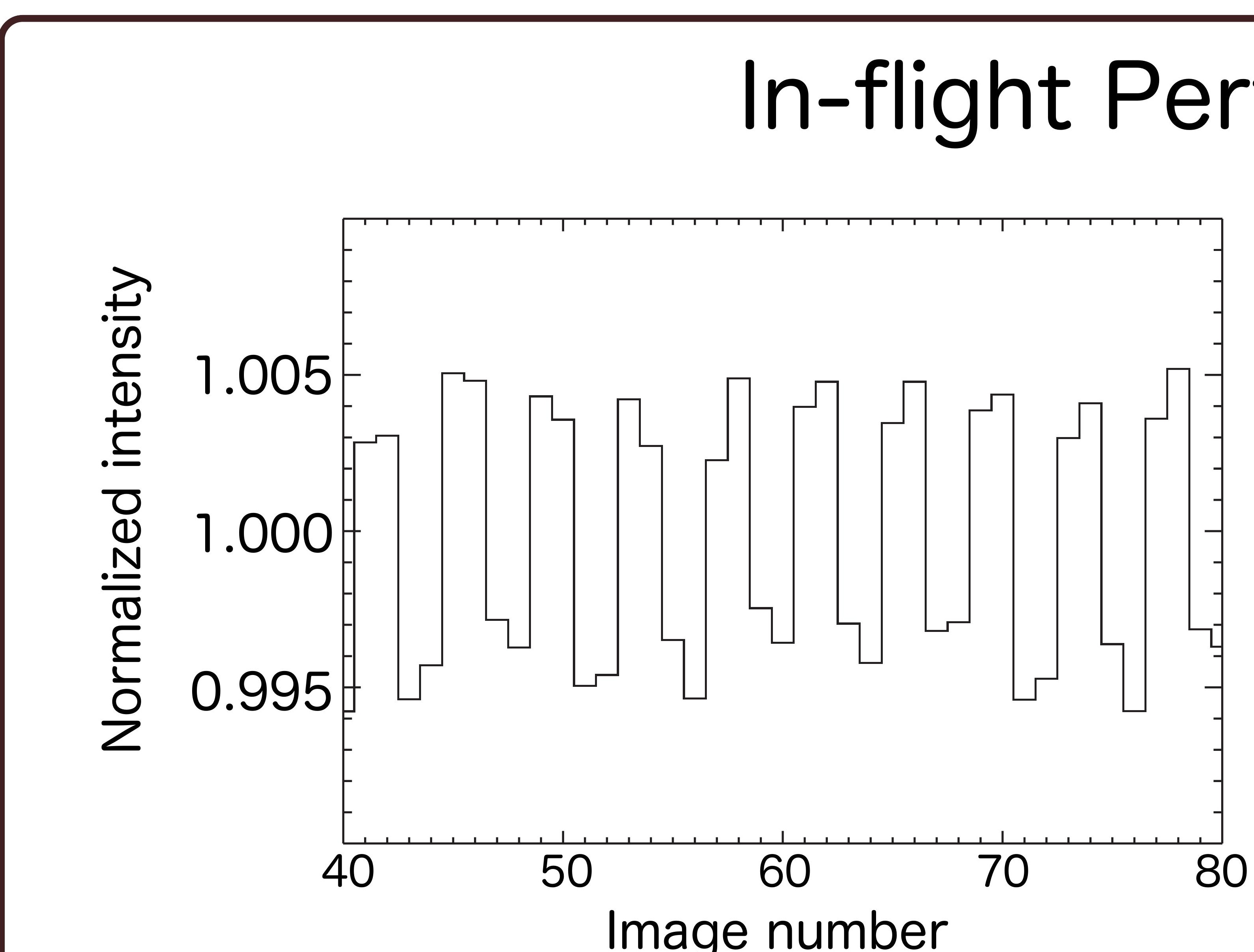

Example of observed modulations (spatially averaged). Local amplitudes are much higher than shown here.
According to the housekeeping data taken during the flight, PMU rotated continuously with no interruption during the whole observational time. The angle error was within the same level as the ground tests, and high rotation uniformity was achieved. Based on this result, we confirmed that CLASP successfully obtained high quality Lyman-alpha polarization data. The CLASP team works for the data analysis and the results will be published soon.

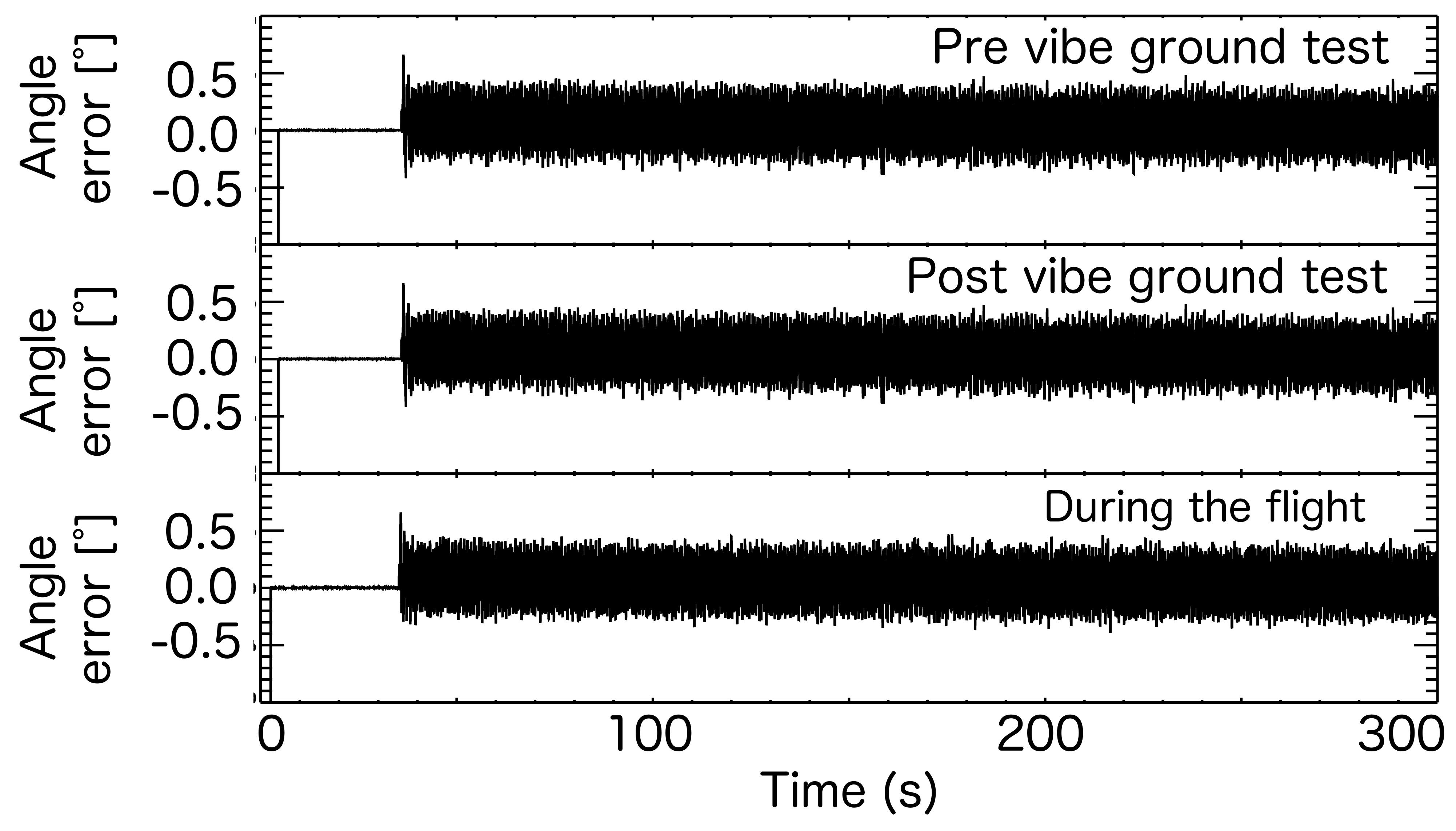

Angle errors during the pre- and post-vibe tests, and the flight 